\title{
Experimental Characterization of Bodycentric Passive RFID Systems.
}

\author{
S.Manzari ${ }^{(1)}$, C. Occhiuzzi ${ }^{(2)}$, G. Marrocco ${ }^{(3)}$ \\ DISP, University of Roma Tor Vergata \\ Via del Politecnico,1, 00133, Roma (ITALY) \\ (1) sabinamanzari@hotmail.it \\ (2) occhiuzzi@disp. uniroma2.it \\ ${ }^{(3)}$ marroccodisp.uniroma2.it
}

\begin{abstract}
Recent progresses in the design of wearable RFID tag antennas stimulate the idea of passive bodycentric systems wherein the required power to drive the wearable tags is directly scavenged from the interrogation signal emitted by the reader unit. While active bodycentric links have been extensively investigated, the feasibility of passive systems is still questionable due to the poor sensitivity of the tags and to the modest read distances. This paper describes an articulated measurement campaign with textile wearable low-profile tags in the UHF RFID band. It is demonstrated that both on-body and off-body links are feasible with a power budget fully compliant with the available technology. The experiments permits to identify the most efficient tag placements and to propose some quantitative and general guidelines useful to characterize and design this kind of new systems.
\end{abstract}

\section{INTRODUCTION}

The RadioFrequency Identification (RFID) technology is increasingly adopted in Logisting, Manufaturing and Security, while pioneristic applications are currently experimented in Sensor Networks, Personal Healthcare and even Entertainment and Social Arts ([1]-[7]). Thanks to the advances in lowpower electronics, battery-less (passive) devices are particularly attractive: in these systems the RF power required to the transponder element (the tag) to respond to the query device (the reader) is scavenged from the interrogation signal by the tag itself. Since passive RFID tags do not require regular recharging, they are particularly suited to disposal usage and to the pervasive and long-term distribution within environments.

In some applications the tag device, comprising an antenna integrated with a microchip transponder and eventually with additional sensors or actuators, needs to be worn onto the human body ([8]-[13]). Tags integrated into clothes could work as a body-centric passive RFID system able to track people's position and/or to monitor life parameters every time and everywhere. Compared with active bodycentric systems, RFID solution could furthermore offer a higher degrees of pervasivity than the active systems due to lower cost and to the absence of local power supply. These kind of systems deserve additional challenges since RFID antennas do not transmit but backscatter the power received by the reader and hence they need to achieve efficient energy scavenging in presence of the very lossy human body while keeping the antenna size small at the involved frequency (UHF: 866-970 MHz including European, US and Asia sub-bands). Up to now, however, the true feasibility and reliability of these class of devices is still questionable, especially concerning the achievable read ranges in real environments in case of real body motion and postures.

As in the more mature active bodycentric systems, interesting RFID communication modalities are on-body and off-body links. The off-body communication could be useful for example to locate and monitor people inside buildings by means of fixed readers placed in different rooms or by a wearable reader and ambient-disseminated tags [21]. A possible application could be the access control in dangerous or restricted areas. The on-body communication is instead typical of unusual scenarios where a fixed communication infrastructure is missing. It could be the case of a sportman or a fireman equipped with different RFID sensors (inside his garment) interrogated by his hand-held standard communicator in harsh environments [22],[23]. Starting from our recent experience with wearable RFID tags ([8],[9],[24]), this paper discusses the feasibility of a bodycentric system including passive RFID textile tags, derived from a previous teflon prototype. Now, an articulated experimental campaign is aimed to understand the achievable read ranges, the minimum number of required tags and their most suitable positions over the body for reliable on-body and off-body links. Activity and shadowing effects will be also taken into account in order to understand the possibility to establish robust and safe communications.

\section{ANTENNA DESIGN AND CHARACTERIZATION}

A $3 \mathrm{~mm}$ thick synthetic felt sample has been chosen as substrate for the wearable antenna. The electromagnetic features of the felt have been experimentally evaluated by means of parameter identification techniques using numerical models of planar test antennas. The achieved permittivity and conductivity at $870 \mathrm{MHz}$, which is the European frequency for RFID systems, are $\epsilon=1.17$ and $\sigma=2 \cdot 10^{-4} \mathrm{~S} / \mathrm{m}$.

The resulting fabricated prototype, of overall size $7 \times 9 \mathrm{~cm}$, is shown in Fig.1. The electromagnetic tag's performances have been experimentally characterized with respect to the 
realized gain, e.g. the radiation gain of the antenna reduced by the impedance mismatch, which directly imposes the tag's read distance. The realized gain, $G_{T} \tau \equiv \hat{G}_{T}$ (Fig.1), is measured by means of the turn-on power method [25], using a a customized UHF long-range reader based on the ThingMagic M5-e ASIC. Especially around $869 \mathrm{MHz}$, simulation and measurements are in a significant agreement, with less than $0.5 d B$ difference.

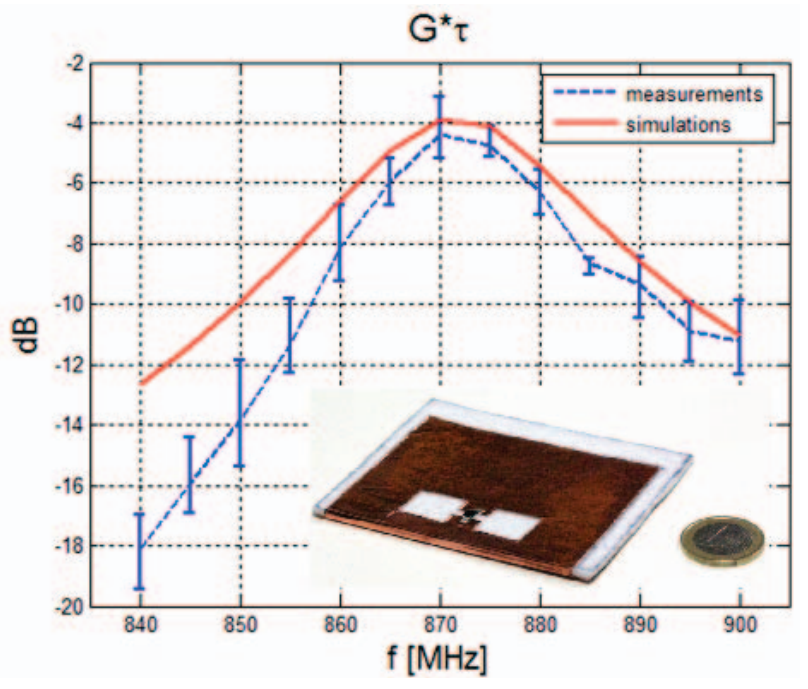

Figure 1. Prototype of body-worn felt antenna matched to $Z$ in $=15-j 135$ $\Omega$ microchip and measured realized gain (along the antenna boresight) for the tag placed on the human torso

\section{EXPERIMENTAL CHARACTERIZATION}

The tag's radiation performances have been experimentally evaluated within a $5.5 m \times 5.5 m \times 3 m$ office room (Fig.2) with desks and computers all around the side walls. The experimental setup included the same reader and antenna as before.

\section{A. Off-body RFID link}

For the characterization of the off-body RFID link, the reader's antenna was placed in the middle of a side wall, $1.3 \mathrm{~m}$ from the floor. The Effective Isotropically Radiated Power EIRP emitted by the reader was $3.2 \mathrm{~W}$, e.g. the maximum value allowed by the European Regulation.

A volunteer, equipped with one or more wearable tags, walked freely within the room with the purpose to test the effective reading range of the proposed tags and to find their minimum number and preferred locations to achieve the maximum coverage of the space independently from the human-reader mutual position. Four different tag placements have been studied (torso, arm, back and shoulders), each in three different polarizations (orientation of the tag).

In most configurations the maximum frontal distance is about $d_{\max }\left(\phi=0^{\circ}\right)=4.5 \mathrm{~m}$, and drastically falls to zero in the rear direction. So, in none of the considered tag placements it is possible to achieve a nearly uniform coverage with just a

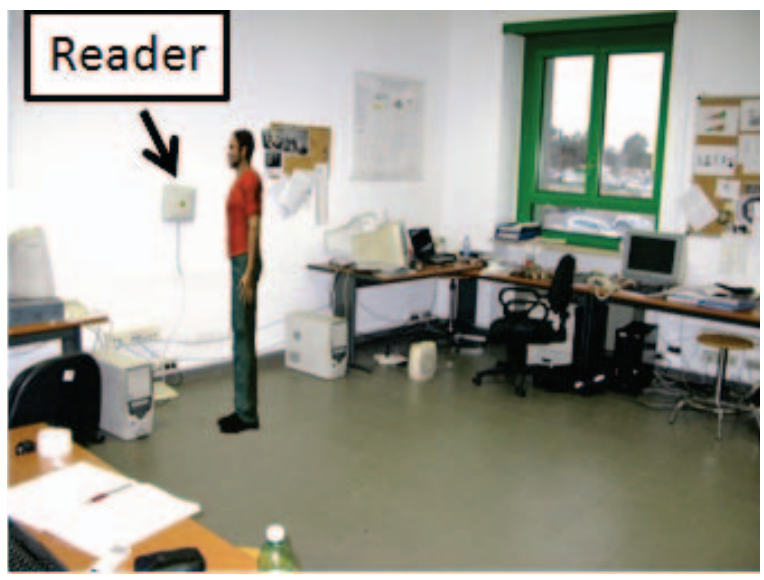

Figure 2. Experimental setup. The volunteer equipped with a wearable tag walks away from the reader'antenna which is placed onto the wall of a $5.5 \mathrm{~m} \times$ $5.5 \mathrm{~m} \times 3 \mathrm{~m}$ room, at $1.3 \mathrm{~m}$ fom the floor. The experiment is repeated for four orientations of the body with respect to the reader's antenna, e.g. for $\phi_{n}=0,90,180,270$ degrees.

single tag. By a combined use of tags it is however expected to achieve a reliable RFID link, for instance by placing one tag over the torso and the other over the back or a single tag over each arm. The combined result for a tag placed on the torso and another one placed on the back is shown in Fig.3 where a nearly isotropic bodycentric interrogation region is visible. This diagram represents the area in which wherever a reader emitting $3.2 \mathrm{~W}$ EIRP in front of the person is placed, it is possible to monitor the subject independently from his position and orientation. Even better results may be achieved by using three tags: over both the arms and one over the chest.

In conclusion, a single reader permits to establish a reliable RFID link with a person equipped with a two- or three-tags within a room of $4 \mathrm{~m}$ by $4 \mathrm{~m}$ size. Four readers, each placed onto each side wall, would instead enable the continuous interrogation within a four-times larger $(9 \mathrm{~m}$ by $9 \mathrm{~m})$ room.

\section{B. On-body RFID link}

The on-body RFID link characterization has been here performed by means of a remotely controlled short range wearable CAEN A528 reader connected to a quarter-lambda patch (PIFA) with maximum gain $3.3 d B$ and placed on the waist, slightly on the left side of the body (Fig.4up).

Five different communication links are considered, with five wearable tags placed on the torso, forearm, head, leg and wrist respectively [26]. During the measurements the volunteer assumes twenty different static and "moving" postures, illustrated in Fig.4down. The reliability of the link is in fact strictly connected to the reader-tag mutual position/orientations and to the body postures.

The RFID link is analyzed according to a statistical approach. The wearable tags are interrogated by means of a fixed power $P_{i n}=20 \mathrm{dBm}$ and the percentages of answer, $\alpha$, defined as

$$
\alpha=\frac{N_{T}}{N_{R}} \times 100
$$




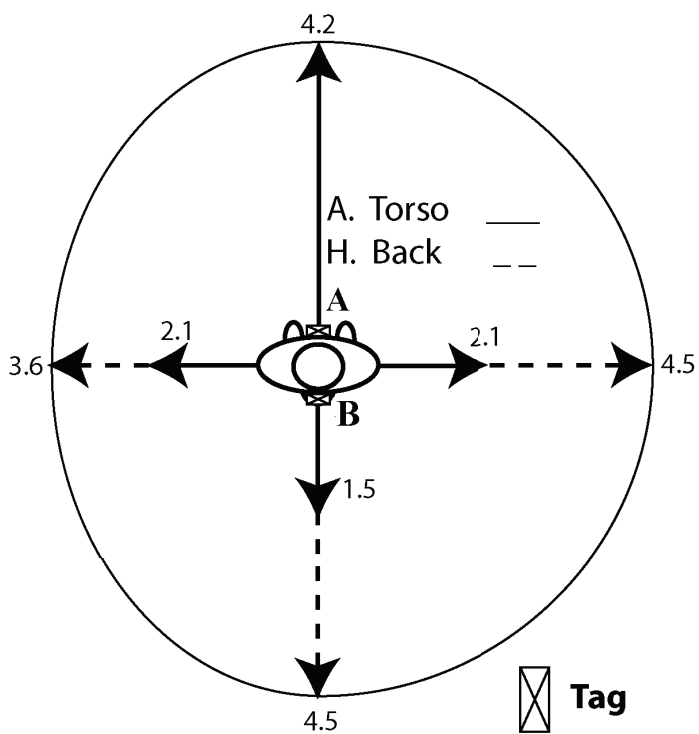

Figure 3. Bodycentric reading regions for two couplet of tags placed on the human body: one tag over the torso (A) and another one over the back (B). Continuous and dashed arrows indicate the maximum read distance $d_{\max }\left(\phi_{n}\right)$ of the two tags in the specific direction and the ellipse-like regions gives the estimate of the overall resulting bodycentric reading region for the combined two-tags system.

is collected for each posture.

The reader performs 9 polls per second, each interrogation period is $10 \mathrm{~s}$ and hence $N_{R}=90$, while $N_{T}$ is the count of responses to those polls.

The aggregated results are shown in Fig. 5 for the particular case of vertical polarization. The waist-torso channel (first row) is the most robust one, with a percentage of answer better than $70 \%$ for all the 20 positions. The other links results more sensitive to the human activity, with lower percentage of answer sometimes close to zero.

\section{CONCLUSION}

The presented experimentations demonstrate that passive bodycentric RFID links are feasible within a regular indoor room with the today available technology. Actually, the onbody link may be established using a query power of the order of just $10 \mathrm{dBm}$, in case of the tag placed over the torso and the reader's antenna on the waist. This small power budget is compatible with pico-readers as well as with conventional hand-held radios and even with smartphones. Moreover, the continuous improvement in microchip sensitivity will permit to extend the reading distance or, conversely, to reduce the required powers. New pervasive applications may be therefore envisaged where low-cost and even disposable wearable tags will interact with multi-services radio devices.

\section{AKNOWLEDGEMENT}

This research has been performed under project "PRIN2008: MultiTag" funded by Italian Ministry of University.
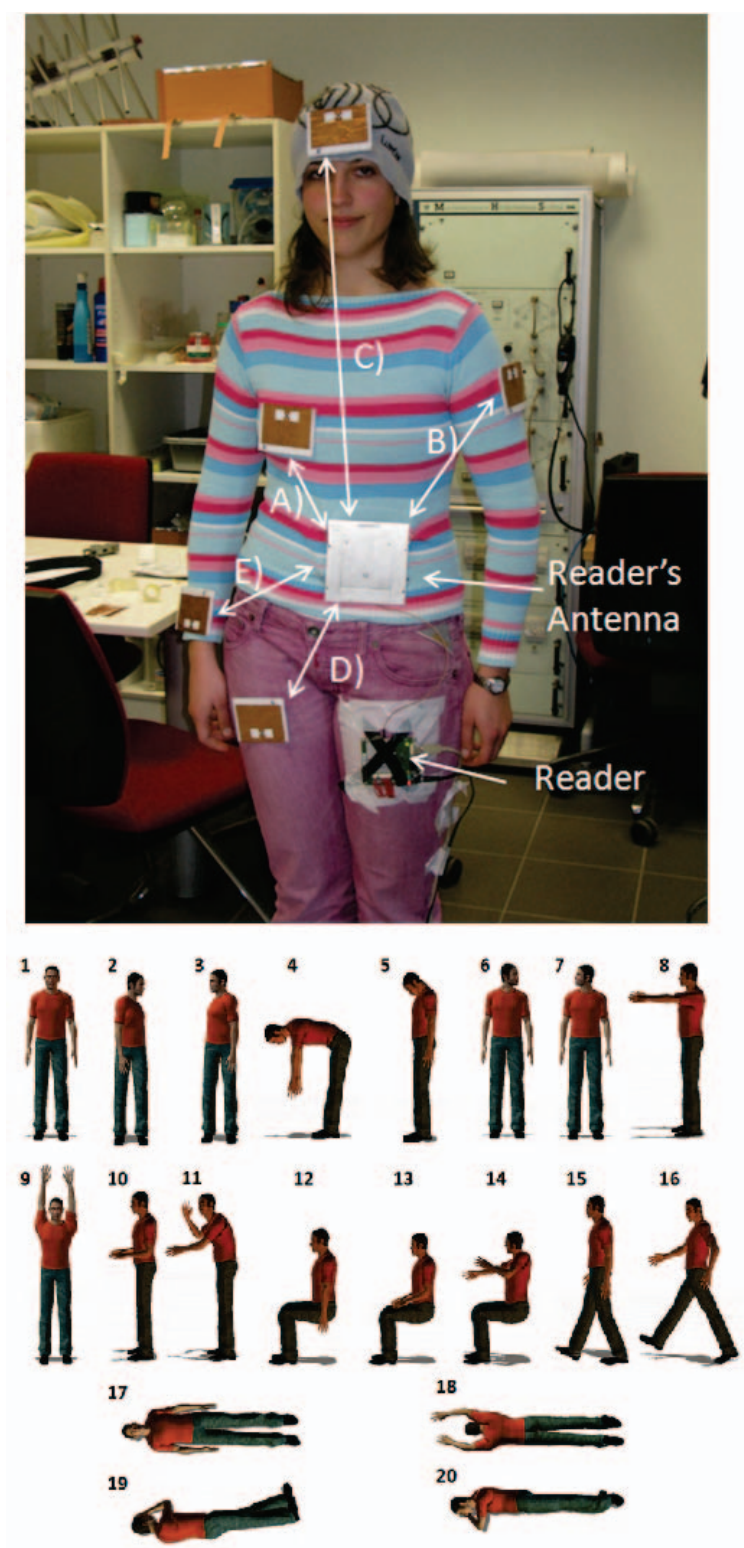

Figure 4. up) Antenna positions on the body. Five different body channels are here considered. The reader is placed in the waist slightly on the left. down) Postures assumed by the subject during the measurements. In the first fourteen the subject stands or sits in different positions according to typical human activities. Two postures (15 and 16) are in motions while in the last four the subjects lies on the floor simulating healthy and pathological conditions, such as the later safety position.

\section{REFERENCES}

[1] S. Jongwoo, T. Sanchez Lopez, K. Daeyoung, "The EPC Sensor Network for RFID and WSN Integration Infrastructure", Fifth Annual IEEE International Conference on Pervasive Computing and Communications Workshops, March 2007.

[2] heng-Ju Li, Li Liu, Shi-Zong Chen, Chi Chen Wu, Chun-Huang Huang, Xin-Mei Chen, "Mobile healthcare service system using RFID Networking”, IEEE International Conference on Sensing and Control, September 2004

[3] L. Cheng-Ju, L. Li, C. Shi-Zong, W. Chi Chen, H. Chun-Huang, and 


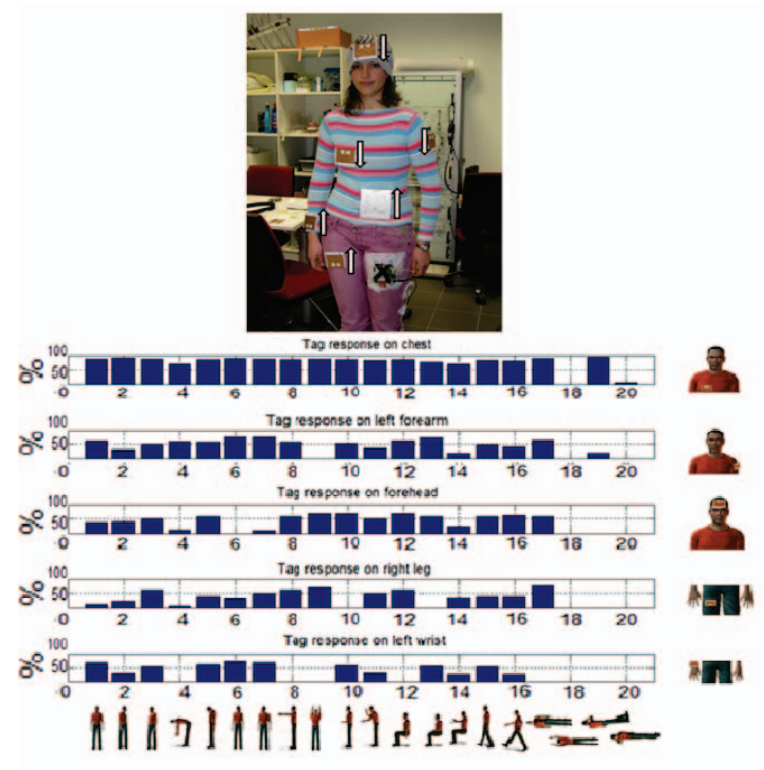

Figure 5. Channel robustness in term of percentage of answer with the antennas in vertical polarization.

C. Xin-Mei, "Mobile healthcare service system using RFID ",IEEE Int.Conf. Networking Sensing and Control, 2004, vol. 2, pp. 1014-1019.

[4] R. S. Sangwan, R. G. Qiu, and D. Jessen,"Using RFID tags for tracking patients, charts and medical equipment within an integrated health delivery network ", IEEE Int. Conf. Networking Sensing and Control, 2004, pp. 1070-1074.

[5] J. Park, J. Seol, Y. Oh, "Design and implementation of an effective mobile healthcare system using mobile and RFID technology", in Proc. 7th Int. Symp. HEALTCOM 2005, pp. 263-266, 2205.

[6] Won Jay Song, S.H. Son, Munkee Choi, Minho Kang, "Privacy and security control architecture for ubiquitous RFID healthcare system in wireless sensor networks", International Conference on Consumer Electronics, January 2006.

[7] O. Rashid, P. Coulton, R. Edwards, W. Bamford; "Utilising RFID for mixed reality mobile games", IEEE International Conference on Consumer Electronics, January 2006.

[8] G. Marrocco, "RFID antennas for the UHF remote monitoring of human subjects", IEEE Trans. Antennas Propagation, Vol.55, N.6, pp. 18621680, June 2007.

[9] C. Occhiuzzi, S. Cippitelli, G. Marrocco, "Modeling, Design and Experimentation of Wearable RFID Sensor Tag ", IEEE Trans. on Antennas \& Propagation, Vol.58, N.8, pp 2490-2498, August 2010.

[10] L.Yang, R.Vyas, A. Rida, J. Pan, M. M. Tentzeris, "'Wearable RFIDEnabled Sensor Nodes for Biomedical Applications"', In proceeding of Electronic Components and Technology Conference Lake Buena Vista, Florida 2008

[11] M. Svanda, M. Polivka, "Extremely Low Profile UHF RFID TAG Antennas for Identification of People", European Conference on Antennas and Propagation EUCAP 2010.

[12] T. Kellomaki,T. Bjorninen, L. Ukkonen, L. Sydanheimo, "Shirt collar tag for wearable UHF RFID systems", European Conference on Antennas and Propagation EUCAP 2010.

[13] Harish Rajagopalan, Y. Rahmat-Samii, "Conformal RFID Antenna Design Suitable for Human Monitoring and Metallic Platforms", European Conference on Antennas and Propagation EUCAP 2010, Barcelona, Spain.

[14] P. S. Hall, Y. Hao, Antennas and Propagation for b.ody-centric wireless communications, 1st Edition, Artech House, INC, Norwood 2006

[15] J. Garcìa, A.Arriola, G. Sasiain, D. Valderas, J.I. Sancho, X. Chen, "Characterization of Phantom Size and Link Budget for Off-Body Communications", European Conference on Antennas and Propagation EUCAP 2010, Barcelona, Spain.
[16] R. Khouri, P. Ratajczak, P. Brachat, R. Staraj, "A Thin SurfaceWave Antenna using a Via-Less EBG structure for $2.45 \mathrm{GHz} \mathrm{ON}$ Body Communication Systems", European Conference on Antennas and Propagation EUCAP 2010, Barcelona, Spain.

[17] L. Vallozzi, W. Vandendriessche, H. Rogier, C. Hertleer, M. Scarpello, "Wearable Textile GPS Antenna For Integration In Protective Garments".

[18] Sankaralingam Subramaniam, Bhaskar Gupta, "A Bluetooth Antenna for On-Body Communications", European Conference on Antennas and Propagation EUCAP 2010, Barcelona, Spain.

[19] A. Sani, A. Alomainy, G. Palikaras, Y. Nechayev, C. Parini, P. S. Hall, "UWB on-body radio propagation and system modelling for wireless body-centric networks", IEEE, Antennas and Propagat., Vol.58, pp. 238241, jan. 2010.

[20] F. Yang, A. Aminian, Y. Rahmat Samii, "A Thin Surface-Wave Antenna using a Via-Less EBG structure for $2.45 \mathrm{GHz}$ ON-Body Communication Systems", European Conference on Antennas and Propagation EUCAP 2010, Barcelona, Spain

[21] G. Marrocco, E. Di Giampaolo, R. Aliberti, "Estimation of UHF RFID Reading Regions in Real Environments", Antennas and Propagation Magazine, IEEE, Vol 51, pp. 44-57,2010.

[22] D. Psychoudakis, G. Y. Lee, C-C. Chen, J. L. Volakis, "Military UHF Body-Worn Antennas for Armored Vests", European Conference on Antennas and Propagation EUCAP 2010.

[23] C. Hertleer, L. Van Langenhove, H. Rogier, L. Vallozzi, A textile antenna for fire fighter garments, AUTEX 2007 Conference (Association of Universities for Textiles): From Emerging Innovations to Global Business (2007) .

[24] C. Occhiuzzi, G. Marrocco, "The RFID Technology for Neurosciences: Feasibility of Limbs' Monitoring in Sleep Diseases" Information Technology in Biomedicine, IEEE Transactions, 2010, Vol. 14, pp. 37 - 43.

[25] G. Marrocco , F. Amato, "Self-sensing Passive RFID: from Theory to Tag Design and Experimentation", Proceedings of the 39th European Microwave Conference.

[26] A. Alomainy, Y. Hao, X. Hu, C. G. Parini, P. S. Hall, "UWB onbody radio propagation and system modelling for wireless body-centric networks", Proc. Inst. Elect. Eng. Commun., vol. 153, pp. 107-114, 2006. J. Ryckaert, P. De Doncker, R. Meys, A. de Le Hoye, and S. Donnay, "Channel model for wireless communication around human body," IEEE Electron. Lett., vol. 40, no. 9, pp. 543-544, Apr. 2004. 